\title{
Electroacupuncture versus sham electroacupuncture for urinary retention in poststroke patients: study protocol for a multicenter, randomized controlled trial
}

Seungwon Shin ${ }^{1}$, Jiwon Lee ${ }^{1}$, Junghee Yoo², Sung Min Lim ${ }^{3^{*}}$ and Euiju Lee $2^{2^{*}}$

\begin{abstract}
Background: This study protocol evaluates the effectiveness of adjuvant electroacupuncture (EA) for urinary retention in poststroke patients undergoing conventional treatments, in comparison with that of a sham control.

Methods/design: A multicenter, blinded, randomized controlled trial will be conducted in three hospitals in the Republic of Korea. We are recruiting 54 stroke survivors (aged $>19$ years), who were diagnosed with urinary retention based on the results of two consecutive post-void residual (PVR) tests, and dividing them randomly into two arms: the EA and Park-sham control groups.

They will receive ten sessions of EA or sham treatment for 2 weeks. The participants will be blinded with nonpenetrating needles and fake sounds of EA stimulators. The daily PVR ratio will be primarily measured at baseline and at the end of the study to statistically test the effectiveness of EA for poststroke urinary retention. Then, the Korean version of the Qualiveen Questionnaire, the Korean version of the International Prostate Symptom Score, and the blinding index will be assessed. After each EA session or sham EA, adverse events will be reported to evaluate the safety of EA.

Results will be analyzed by using the independent $t$-test or Mann-Whitney $U$ test, based on both intention-to-treat and per-protocol principles.
\end{abstract}

Discussion: The findings will provide clinical evidence for the effectiveness of EA treatment to improve urinary retention in stroke survivors.

Trial Registration: This study protocol was registered in ClinicalTrials.gov (NCT02472288) on 10 June 2015.

Keywords: Stroke, Cerebrovascular accident, Urinary retention, Electroacupuncture, Randomized Controlled Trial

\section{Background}

Urinary retention (UR) or incomplete bladder emptying (IBE), defined as a post-void residual (PVR) urine volume of $>100 \mathrm{~mL}$ on two consecutive occasions [1,2], occurs in approximately $29 \%$ [1] to $56 \%$ [3] of poststroke patients. Bladder problems that occur after stroke episodes deteriorate the stroke survivors' quality of life (QoL) and affect

\footnotetext{
* Correspondence: limsm@outlook.kr; sasangin@daum.net

${ }^{3}$ Department of Motor \& Cognition Rehabilitation, Korean National

Rehabilitation Research Institute, 111 Gaorigil, Gangbuk-gu, Seoul 01022,

Republic of Korea

${ }^{2}$ College of Korean Medicine, Kyung Hee University, 23 Kyungheedae-ro,

Dongdaemun-gu, Seoul 02447, Republic of Korea

Full list of author information is available at the end of the article
}

their disability and mortality [4-6]. UR in stroke survivors is usually managed with catheterization, but this procedure interferes with their active rehabilitation and can induce urinary tract infection (UTI) [2]. In the long term, UR without adequate management can lead to renal dysfunction [7]. Thus, caregivers should provide poststroke patients who have UR with proper bladder management to improve the patients' functional outcomes [8].

In the United Kingdom, sacral neurostimulation has been known to be effective for UR induced by urethral sphincter overactivity (Fowler's syndrome). However,

\section{$\int$ Biomed Central}

(c) 2016 Shin et al. Open Access This article is distributed under the terms of the Creative Commons Attribution 4.0 International License (http://creativecommons.org/licenses/by/4.0/, which permits unrestricted use, distribution, and reproduction in any medium, provided you give appropriate credit to the original author(s) and the source, provide a link to the Creative Commons license, and indicate if changes were made. The Creative Commons Public Domain Dedication waiver (http://creativecommons.org/publicdomain/zero/1.0/) applies to the data made available in this article, unless otherwise stated. 
significant complications have occurred, such as inserted lead migration or high surgical revision rates [9]. Bladder problems have also been a concern of traditional medicine practitioners. Diabetic bladder dysfunction was improved with acupuncture treatment in a pilot study [10]. Another randomized controlled trial showed that, besides sham electroacupuncture (EA), EA is an effective treatment of bladder muscle overactivity in poststroke patients [11]. A small-scale of clinical study with a single study group suggested the potential benefit of EA therapy for poststroke patients with IBE.

However, randomized controlled trials designed with adequate statistical power and sham comparators should be conducted to determine whether EA is effective for poststroke UR. We will conduct a multicenter, randomized controlled trial to evaluate the effectiveness of adjuvant EA therapy for poststroke patients with UR who are undergoing conventional treatments, in comparison with that of sham EA.

\section{Methods/design \\ Study design}

This is a multicenter, blinded, randomized controlled trial to compare an EA group with a sham EA group, with an allocation ratio of 1:1. We designed the protocol, conforming to the Consolidated Standards of Reporting Trials (CONSORT) [12] and Standards for Reporting Interventions in Clinical Trials of Acupuncture (STRICTA) [13] guidelines. The study process is illustrated in Fig. 1. The institutional review board of Kyung Hee University Korean Medicine Hospital approved the study (KOMCIRB150213-HR-005). The protocol has been registered in ClinicalTrial.gov (NCT02472288).

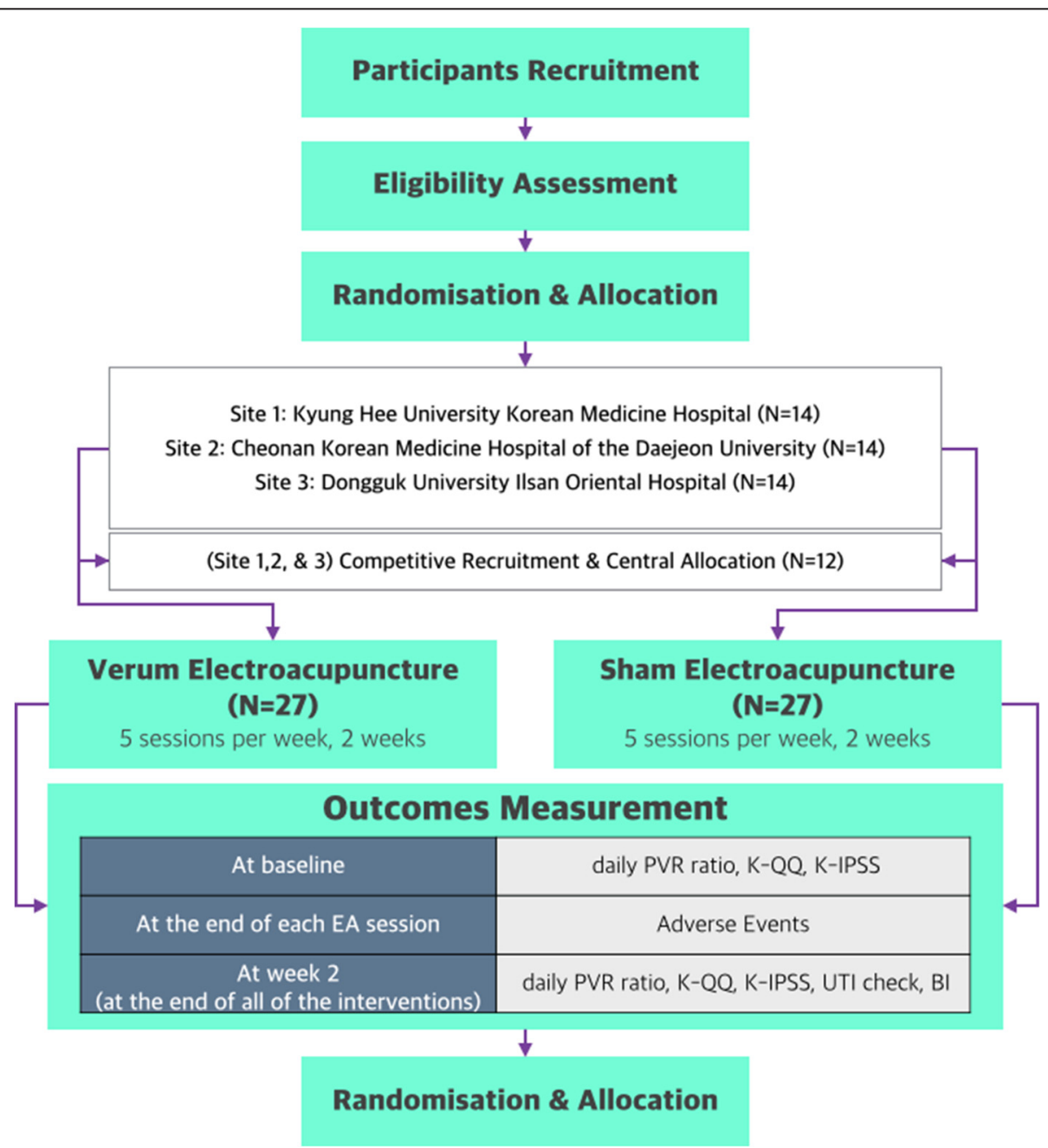

Fig. 1 Flowchart of the study process. Fifty-four participants will be randomized and allocated to either the intervention or control group. After conducting ten sessions of verum or sham electroacupuncture for 2 weeks and examining for urinary tract infection (UTI), the daily post-void residual (PVR) ratio, Korean version of Qualiveen Questionnaire score (K-QQ), Korean version of the International Prostate Symptom Score (K-IPSS), and blinding index (BI) will be measured. Adverse events will be investigated right after each session 


\section{Participants}

We will recruit 54 male or female patients aged $\geq 19$ years from three hospitals in Korea: Kyung Hee Korean Medicine Hospital in Seoul, Cheonan Korean Medicine Hospital of Daejeon University in Cheonan, and Dongguk University Ilsan Oriental Hospital in Ilsan. In order to identify survivors from stroke episodes, like intracerebral hemorrhage or cerebral infarction, experienced practitioners will check the PVR urine volume twice a day. The patients will be pre-screened only if both residual volumes are $>100 \mathrm{~mL}$. After we obtain informed consent from the patients or their representatives, we will screen them according to the eligibility criteria for enrollment in the study.

\section{Inclusion criteria}

We will include both male and female patients aged $>19$ years whose stroke episodes occurred within the last 2 years. We will not restrict inclusion of participants according to type of stroke or damaged region in the brain. Stroke history will be confirmed based on computed tomography or magnetic resonance imaging examination results. Two consecutive PVR tests will be performed, and the patient with the results of both residuals > $100 \mathrm{~mL}$ will be included in the trial $[1,2]$.

\section{Exclusion criteria}

We will exclude stroke patients with any one of the following clinical conditions from the medical screening process: (1) bleeding disorders, (2) procedures or surgeries for peripheral vascular diseases, (3) psychiatric disorders, (4) severe diseases in the lower urinary tract, (5) acute or chronic infectious diseases in the lower urinary tract, or (6) pregnancy (laboratory results from urinalysis). In addition, patients (7) whose medications for UR or relevant symptoms (urinary incontinence drugs or diuretics) have been changed within 3 days or (8) for whom onset of treatment is within 1 week prior to the study, along with those with a Glasgow Coma Scale score of $<8$ points, will be excluded. Finally, we will also exclude those who have fears about acupuncture therapy.

\section{Dropout criteria}

Patients will be removed from the study if they are unwilling to continue their participation in the study. Patients who fail or anticipate to fail in continuing at least seven sessions will be dropped from the study, too.

\section{Randomization}

Random sequences were generated separately in each site with the $\mathrm{R}$ package ( $\mathrm{R}$ Foundation for Statistical Computing, Vienna, Austria, https://www.r-project.org) to allocate participants into either the EA or sham group. The allocation ratio was 1:1. From each center, at least 14 participants will be recruited, and the last 12 persons will be competitively enrolled among all the three sites. Only the delegated sub-investigators can access the security electronic file of the randomization table and assign the participants. Practitioners from each site will call or send a message to the sub-investigators in order to allocate the participants in their own hospitals. After assignments are completed, a sub-investigator from the primary site, Kyung Hee University Korean Medicine Hospital, will allocate the 12 participants from the three sites to the last participant. The investigators, who will generate the random number and assignments, will be blinded to the arm where each participant will be allocated.

\section{Blinding}

The patients and outcome assessors will remain blinded during the study. Patients assigned to the sham group will undergo a similar treatment as those assigned to the EA group. Because we will use the Park-sham with nonpenetrating acupuncture needles as control, electrostimulation will be turned on without conductance. In addition, we will use the same guide tubes used in the Park-sham acupuncture needles in the intervention group, too. Aside from the practitioners who will administer the EA or sham treatment, there will be an independent outcome assessor who will not be notified about the patients' allocated arms.

\section{Interventions}

The EA and sham EA regimens are based on traditional Korean medicine. We referred to the textbook [14] and consulted acupuncture and stroke specialists.

The participants allocated to the EA group will receive five sessions per week of EA therapy for 2 weeks (ten sessions in total). After inserting needles (stainless steel, $0.25 \mathrm{~mm}$ in diameter and $4.0 \mathrm{~mm}$ in length; Dong Bang Acupuncture Inc., Republic of Korea) using the Parksham guide tube on bilateral acupoints BL31, BL32, BL33, and BL34 (eight points in total) at $5-10 \mathrm{~mm}$ in depth, the de qi sensation is elicited. The locations of the acupoints according to the World Health Organization Standard Acupuncture Point Locations in the Western Pacific Region are illustrated in Fig. 2 [15]. De qi means an acupuncture needle sensation of soreness, tingling, fullness, ache, coolness, warmth, and heaviness, and a radiating sensation at and around the acupoints to the point of a threshold that elicits nerve impulse transmission to the cerebral cortex [16]. Then, electrostimulation will be administered for 20 minutes at a moderate frequency of $30 \mathrm{~Hz}$ (STN-111, Stratek, Republic of Korea).

We will use the Park-sham device in the control group. The non-penetrating needles of the sham acupuncture will be implemented on the same eight bilateral acupoints. The total number of sessions of sham EA is ten (five times 


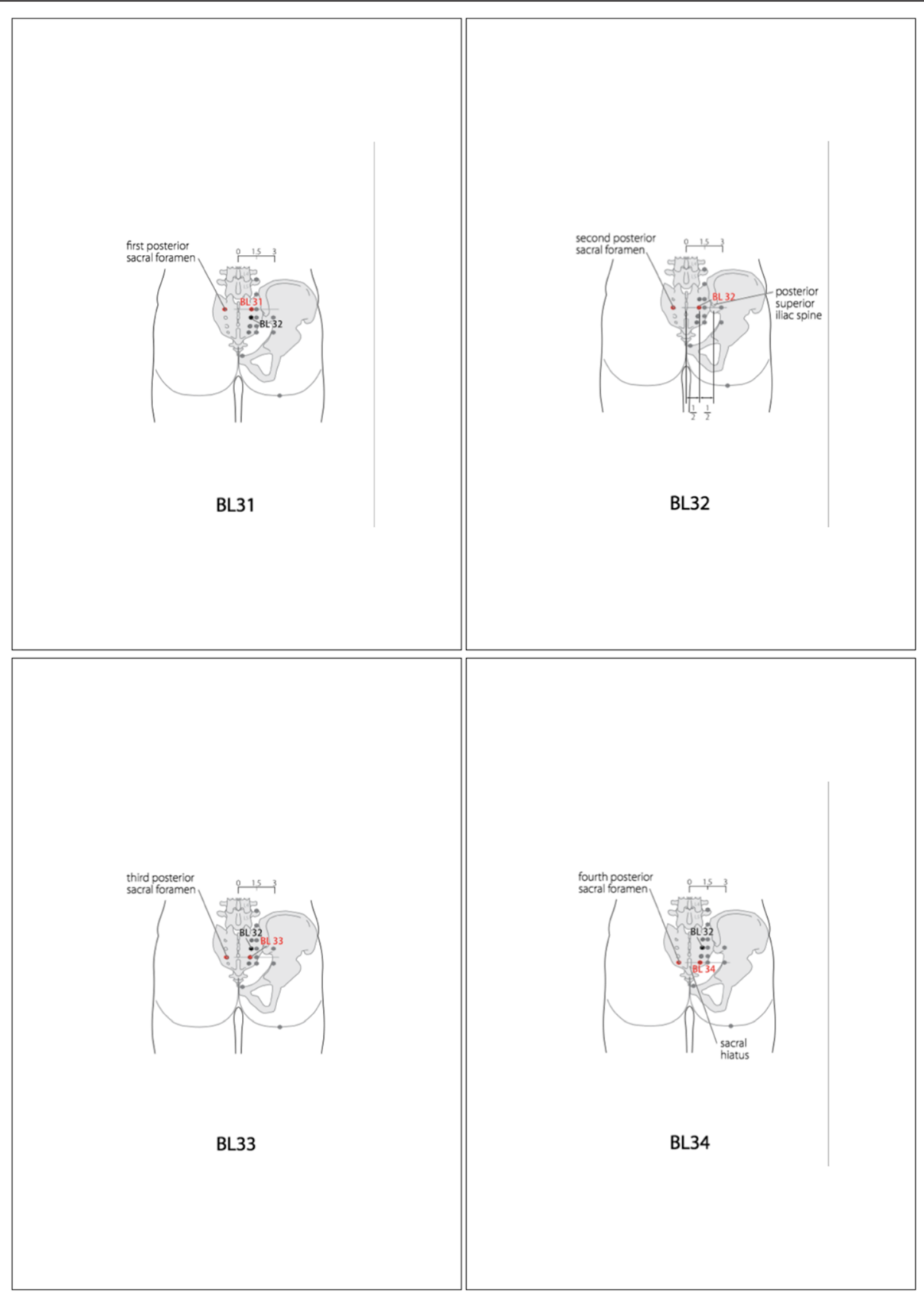

Fig. 2 The locations of acupoints BL31, BL32, BL33, and BL34. In the sacral region, BL31, BL32, BL33, and BL34 are located in the first, second, third, and fourth posterior sacral foramens, respectively. (World Health Organization Standard Acupuncture Point Locations in the Western Pacific Region)

per week for 2 weeks). The electrostimulation will also be administered for 20 minutes at moderate frequency $(30 \mathrm{~Hz}$; STN-111, Stratek, Republic of Korea), even though the stimulation will not be delivered through the skin because of the non-penetrating needle of sham device.

The use of conventional therapies will be allowed during the study for both groups. The conventional therapies consist of general supportive care, preventive or therapeutic management for medical/neurological complications, or drug treatments with antiplatelet agents, anticoagulants, or neuroprotectants. Moreover, stroke management with traditional herbal medications, acupuncture without electrostimulation, or rehabilitation for stroke complications will be permitted during the trial. However, any drugs, herbal medications, or rehabilitation that could affect UR will be prohibited. The study will 
conform to clinical practice guidelines for stroke management in Korea [17].

Practitioners who have over 1 year of clinical experience will perform the EA or sham EA treatments.

\section{Primary outcome}

To evaluate the effectiveness of EA treatment over the sham device for poststroke UR, we will primarily assess the change in daily PVR urine ratios between the baseline and end point of the last session. The daily PVR ratio is computed by dividing the daily PVR urine volume by the daily PVR urine volume plus spontaneous voiding volume. The daily PVR urine volume for 24 hours will be examined with ultrasonography scanning. If the PVR urine volume is more than $100 \mathrm{~mL}$, the residual will be removed through intermittent cauterization before the scanning. When spontaneous urination is impossible, a 24-hour weight change in diaper will be measured for the self-voiding volume.

If the urinary tract is infected at the end, the outcome assessor will measure the PVR urine volume on the third day of the last session after anti-UTI treatment for 48 hours. Otherwise, PVR urine volume will be measured on the next day of the last session.

\section{Secondary outcome}

Because UTI might affect the urine residuals [18, 19], the patients in both groups will undergo urinalysis to determine whether UTI exists before PVR urine volume evaluation at the end point. UTI is diagnosed when nitrite is detected by urinalysis and two of the following clinical symptoms exist: pyuria, lower abdominal pain, or hematuria [20]. The urinalysis retest is performed after 48 hours of antibiotics treatment.

We will also assess disease-specific QoL by using the Korean version of the Qualiveen Questionnaire (K-QQ). This is an assessment tool consisting of 30 items for patients with neurogenic bladder [21-24]. Each item, rated from 0 to 4 points, evaluates patients' bladder problems, while the additive nine questions, rated from -2 to +2 points, are about patients' general QoL status. The higher the absolute value of the total K-QQ score, the lower the QoL becomes. We will measure the K-QQ scores at baseline and at the end of the last session.

The Korean version of the International Prostate Symptom Score will be assessed at baseline and at the end point. This scale is generally used to evaluate general symptoms related to the lower urinary tract $[25,26]$. The total score $(0-35)$ is calculated by seven items of $0-5$ points each, and the severity will be assessed as mild (0-7 points), moderate (8-19 points), or severe (20-35 points).

The frequencies of urination and urinary incontinence will also be reported. The patients will record them by urinary diary for 1 day before the first intervention and for another day after the last intervention. The mean change from posttreatment to pretreatment in the EA group will be compared with that in the sham group.

Finally, we will measure the blinding index (BI) for all of the participants at the end of the verum or the sham EA. The success or failure of the participants' blinding will be assessed by using the validated scale [27]. The outcome assessor will ask the participants where they belong by selecting one of the following responses: "I think I belonged to the EA group," "I think I belonged to the sham group," or "I don't know which group I belonged to."

The patient-rated outcomes will not be measured when the participants are unconscious. The two caregivers with more than 1 year of practical experience, after being educated about the scales, will independently fill in the scales for the participants with low cognitive function and the discrepancy will be settled through discussion.

\section{Other outcomes}

Information on diabetes mellitus (DM) and benign prostatic hyperplasia $(\mathrm{BPH})$ will be obtained from the participants' medical history.

\section{Safety assessment}

Adverse events will be reported to evaluate the safety of EA for poststroke patients with UR. The questionnaires will be administered to the participants after every verum or sham EA session, and the results will be recorded and analyzed. The outcome assessors will judge the severity (mild, moderate, or severe), seriousness, and causality (definitely related, probably related, possibly related, possibly not related, definitely not related to the intervention, or not assessable).

\section{Sample size}

Based on a previous study [2], the mean percentage change in PVR ratios in the EA and control groups were computed. We assumed an after-treatment PVR ratio of 0.65 in the control group, as it was not reported in the study. The null hypothesis was that the percentage changes in PVR ratios have no significant difference between the intervention and control groups. With a significance level of 0.05 and a statistical power of 0.8 , the calculated sample size in total was 42 [28]. Considering a $20 \%$ drop rate at a 1:1 allocation ratio, we will recruit 27 participants for each group.

\section{Data analysis}

We will analyze results primarily based on the principle of intention - to - treat (ITT) and subordinately based on the per - protocol (PP) principle. The ITT analysis will include any participants who are randomly allocated and have at least one EA or sham session. Missing 
values will be imputed by the last observation carried forward. The PP analysis will cover only the patients who have at least seven sessions of EA or sham treatments and have no medical diagnosis of UTI at the end point.

Continuous variables will be tested by the independent $t$-test if normality is obtained. Otherwise, the MannWhitney $U$ test will be implemented. The categorical variables will be analyzed by the Pearson $\chi^{2}$ test or Fisher's exact test.

\section{Discussion}

This randomized controlled trial aims to determine whether EA treatment is effective for UR in poststroke survivors. The effectiveness is represented by the relative proportion of urine residuals and voiding volumes. Because this study is designed to provide clinical evidence for the relevant patients and practitioners, we have chosen not to eliminate any other general treatments for poststroke survivors.

The eight acupoints were identified based on the guideline published by the World Health Organization. In the sacral region, BL31, BL32, BL33, and BL34 are located in the first, second, third, and fourth posterior sacral foramens, respectively [15]. In the literature on traditional Korean medicine, these points have been reported to possibly have the property to recover bladder dysfunction, and they have shown clinical effectiveness for poststroke detrusor overactivity in a previous study [11]. The acupoints are considered to have a possible mechanism that the acupuncture stimulation directly increases the excitability of the pelvic nerve, which consequently innervates the detrusor muscle [2].

Park-sham acupuncture is a placebo acupuncture device invented to stabilize the needle in position with an additional guide tube preventing skin penetration [29]. This sham device was validated to be inactive and indistinguishable [30]. We have decided to use the same guide tubes as Park-sham acupuncture to prevent participants from discovering the group to which they belong. The apparatus for electrostimulation will be activated in the sham control group to make the participants hear the same machine sound so as to let them believe they are under real acupuncture treatment. Because Parksham acupuncture does not intrude the skin, electrostimulation will not be conducted through the body. Success or failure of these blinding strategies will be appraised at the end of the study by the BI [26].

Bladder dysfunction, including UR, poses an obstacle for rehabilitation and QoL improvement in poststroke patients $[2,4-6]$. Catheterization, the major therapeutic approach, confers the risk of UTI [2]. A preliminary clinical study showed the potential benefits and safety of EA treatment for stroke patients with UR [2]. However, the study was performed only with a single group and lacked the methodological requirements and statistical power to establish the evidence for treatment. Overall, clinical trials of EA for any urinary dysfunction, with a placebo control, are rare, except for the study of poststroke detrusor overactivity [11]. We targeted UR in poststroke patients and designed a randomized, sham-controlled trial to be conducted in multiple sites to appropriately generalize the results of the trial, following the CONSORT [12] and STRICTA guidelines [13].

At the end of the project, we expect that the findings will provide clinical evidence of the effectiveness of EA treatment for improving UR in stroke survivors.

\section{Abbreviations \\ BI: blinding index; $\mathrm{BPH}$ : benign prostatic hyperplasia; DM: diabetes mellitus; EA: electroacupuncture; IBE: incomplete bladder emptying; ITT: intention to treat; K-QQ: Korean version of Qualiveen Questionnaire; PP: per protocol; PVR: post-void residual; QoL: quality of life; UR: urinary retention; UTI: urinary tract infection.}

\section{Competing interests}

The authors declare that they have no competing interests.

\section{Authors' contributions}

EL conceived of the project, obtained the funding, developed the trial protocol, and is in charge of the whole implementation process. SML developed the research question, set the hypothesis, and helped to develop the protocol. SS and JY revised the protocol critically, and SS wrote the initial draft of the manuscript. $J$ is in charge of the recruitment of participants and the arrangement of the whole study process. All of the authors read and approved the final manuscript.

\section{Authors' information}

SS (Korean medicine doctor) and $J$ (Korean medicine doctor) are affiliated with Department of Clinical Korean Medicine, Graduate School, Kyung Hee University. JY (nurse, Ph.D. in Korean Medicine) and EL (Korean medicine doctor, Ph.D. in Korean Medicine, professor) are affiliated with College of Korean Medicine of Kyung Hee University, Republic of Korea. EL, a clinical professor, and $J L$ work in the Stroke Center of Kyung Hee University Korean Medicine Hospital. SML (Korean medicine doctor, Ph.D. in Korean Medicine) is a staff member of the Department of Motor and Cognition Rehabilitation, Korean National Rehabilitation Research Institute, Republic of Korea. SS is a research physician at the Korean Medicine Clinical Trial Center in Kyung Hee University Korean Medicine Hospital.

\section{Acknowledgments}

This research was supported by the R\&D grant (2015008) on rehabilitation by the Korea National Rehabilitation Center Research Institute, Ministry of Health \& Welfare.

\section{Author details \\ ${ }^{1}$ Department of Clinical Korean Medicine, Graduate School, Kyung Hee University, 23 Kyungheedae-ro, Dongdaemun-gu, Seoul 02447, Republic of Korea. ${ }^{2}$ College of Korean Medicine, Kyung Hee University, 23 \\ Kyungheedae-ro, Dongdaemun-gu, Seoul 02447, Republic of Korea. ${ }^{3}$ Department of Motor \& Cognition Rehabilitation, Korean National Rehabilitation Research Institute, 111 Gaorigil, Gangbuk-gu, Seoul 01022, Republic of Korea.}

Received: 24 June 2015 Accepted: 29 March 2016

Published online: 12 April 2016

\section{References}

1. Kong KH, Young S. Incidence and outcome of poststroke urinary retention: a prospective study. Arch Phys Med Rehabil. 2000;81:1464-7. 
2. Yu KW, Lin CL, Hung CC, Chou EC, Hsieh YL, Li TM, et al. Effects of electroacupuncture on recent stroke inpatients with incomplete bladder emptying: a preliminary study. Clin Interv Aging. 2012;7:469-74.

3. Garrett VE, Scott JA, Costich J, Aubrey DL, Gross J. Bladder emptying assessment in stroke patients. Arch Phys Med Rehabil. 1989;70:41-3.

4. Anderson CS, Jamrozik KD, Broadhurst RJ, Stewart-Wynne EG. Predicting survival for 1 year among different subtypes of stroke. Results from the Perth Community Stroke Study. Stroke. 1994;25:1935-44.

5. Taub NA, Wolfe CD, Richardson E, Burney PG. Predicting the disability of first-time stroke sufferers at 1 year. 12-month follow-up of a populationbased cohort in southeast England. Stroke. 1994;25:352-7.

6. Patel M, Coshall C, Rudd AG, Wolfe CD. Natural history and effects on 2-year outcomes of urinary incontinence after stroke. Stroke. 2001;32:122-7.

7. Mustonen S, Ala-Houhala IO, Tammela TL. Long-term renal dysfunction in patients with acute urinary retention. Scand J Urol Nephrol. 2001;35:44-8.

8. Mizrahi EH, Waitzman A, Arad M, Blumstein T, Adunksy A. Bladder management and the functional outcome of elderly ischemic stroke patients. Arch Gerontol Geriatr. 2011;53:e125-8.

9. Datta SN, Chaliha C, Singh A, Gonzales G, Mishra VC, Kavia RB, et al. Sacral neurostimulation for urinary retention: 10-year experience from one UK centre. BJU Int. 2008:101:192-6.

10. Tong Y, Jia Q, Sun Y, Hou Z, Wang Y. Acupuncture in the treatment of diabetic bladder dysfunction. J Altern Complement Med. 2009;15:905-9.

11. Liu Y, Liu L, Wang X. Electroacupuncture at points Baliao and Huiyang (BL35) for post-stroke detrusor overactivity. Neural Regen Res. 2013:8:1663-72

12. Schulz KF, Altman DG, Moher D, CONSORT Group. CONSORT 2010 Statement: updated guidelines for reporting parallel group randomised trials. BMC Med. 2010;8:18.

13. MacPherson $H$, Altman DG, Hammerschlag R, Youping L, Taixiang W, White A, et al. Revised STandards for Reporting Interventions in Clinical Trials of Acupuncture (STRICTA): extending the CONSORT statement. PLoS Med. 2010;7:e1000261.

14. Korean Acupuncture \& Moxibustion Society. Acupunture \& Moxibustion. Seoul: Jipmudang; 2008

15. WHO Regional Office for the Western Pacific. WHO standard acupuncture point locations in the Western Pacific Region. Manila: World Health Organization; 2008.

16. Xie H, Li X, Lai J, Zhou Y, Wang C, Liang J. Effectiveness of De Qi during acupuncture for the treatment of tinnitus: study protocol for a randomized controlled trial. Trials. 2014:15:397.

17. Clinical Research Center for Stroke. Clinical practice guidelines for stroke (revision 2015.03.). Seoul: Clinical Research Center for Stroke; 2015.

18. Stern JA, Hsieh YC, Schaeffer AJ. Residual urine in an elderly female population: novel implications for oral estrogen replacement and impact on recurrent urinary tract infection. J Urol. 2004;171:768-70.

19. Truzzi JC, Almeida FM, Nunes EC, Sadi MV. Residual urinary volume and urinary tract infection-when are they linked? J Urol. 2008;180:182-5.

20. Rowe TA, Juthani-Mehta M. Diagnosis and management of urinary tract infection in older adults. Infect Clin North Am. 2014;28:75-89.

21. Bonniaud V, Bryant D, Parratte B, Gallien P, Guyatt G. Qualiveen: a urinary disorder-specific instrument for use in clinical trials in multiple sclerosis. Arch Phys Med Rehabil. 2006;87:1661-3.

22. Bonniaud V, Parratte B, Amarenco G, Jackowski D, Didier JP, Guyatt G. Measuring quality of life in multiple sclerosis patients with urinary disorders using the Qualiveen questionnaire. Arch Phys Med Rehabil. 2004;85:1317-23.

23. Costa P, Perrouin-Verbe B, Colvez A, Didier J, Marquis P, Marrel A, et al. Quality of life in spinal cord injury patients with urinary difficulties. Development and validation of Qualiveen. Eur Urol. 2001;39:107-13.

24. Lee YS, Kim ES, Oh SJ, Lee BS, Kim DA. The linguistic validation and reliability of the Korean version "Qualiveen questionnaire". J Korean Acad Rehabil Med. 2010;34:524-43.

25. Sagnier PP, MacFarlane G, Richard F, Botto H, Teillac P, Boyle P. Results of an epidemiological survey using a modified American Urological Association symptom index for benign prostatic hyperplasia in France. J Urol. 1994;151:1266-70.

26. Choi HR, Chung WS, Shim BS, Kwon SW, Hong SJ, Chung BH, et al. Translation validity and reliability of I-PSS Korean version. Korean J Urol. 1996;37:659-65.
27. Bang HJ, Ni L, Davis CE. Assessment of blinding in clinical trials. Control Clin Trials. 2004;25:143-56.

28. Fleiss JL, Levin B, Paik MC. Statistical methods for rates and proportions. 3rd ed. Hoboken: John Wiley \& Sons; 2013.

29. Park JB, White A, Lee HJ, Ernst E. Development of a new sham needle. Acupunct Med. 1999;17:110-2.

30. Park JB, White A, Stevinson C, Ernst E, James M. Validating a new nonpenetrating sham acupuncture device: two randomised controlled trials. Acupunct Med. 2002;20:168-74.

\section{Submit your next manuscript to BioMed Central and we will help you at every step:}

- We accept pre-submission inquiries

- Our selector tool helps you to find the most relevant journal

- We provide round the clock customer support

- Convenient online submission

- Thorough peer review

- Inclusion in PubMed and all major indexing services

- Maximum visibility for your research

Submit your manuscript at www.biomedcentral.com/submit
) Biomed Central 